\title{
Generating Quitline calls during Australia's National Tobacco Campaign: effects of television advertisement execution and programme placement
}

\author{
T Carroll, B Rock
}

Tobacco Control 2003;12(Suppl II):ii40-ii44

See end of article for authors' affiliations

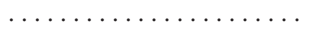

For correspondence: T Carroll, Australian Department of Health and Ageing, GPO Box 9848, Sydney 2001, NSW

Australia;

tom.carroll@health.gov.au of different television advertisements and Objective: The study sought to measure the relative efficiency of different television advertisements and
types of television programmes in which advertisements were placed, in generating calls to Australia's national Quitline.

Design: The study entailed an analysis of the number of calls generated to the Quitline relative to the weight of advertising exposure (in target audience rating points (TARPs) for particular television advertisements and for placement of these advertisements in particular types of television programmes. A total of 238 television advertisement placements and 1769 calls to the Quitline were analysed in Sydney and Melbourne.

Results: The more graphic "eye" advertisement conveying new information about the association between smoking and macular degeneration leading to blindness was more efficient in generating quitline calls than the "tar" advertisement, which reinforced the message of tar in a smoker's lungs. Combining the health effects advertisements with a quitline modelling advertisement tended to increase the efficiency of generating Quitline calls. Placing advertisements in lower involvement programmes appears to provide greater efficiency in generating Quitline calls than in higher involvement programmes.

Conclusions: Tobacco control campaign planners can increase the number of calls to telephone quitlines by assessing the efficiency of particular advertisements to generate such calls. Pairing of health effect and quitline modelling advertisements can increase efficiency in generating calls. Placement of advertisements in lower involvement programme types may increase efficiency in generating Quitline calls.
$\mathrm{T}$ he utilisation of telephone quitline services to assist smokers through the quitting process has been reported as a common feature of comprehensive tobacco control programmes in England, Scotland, Australia, The Netherlands, and parts of the United States. ${ }^{1}$ Studies have identified that callers to these services often represent heavier smokers with greater needs for assistance in quitting ${ }^{2-4}$ and that such services achieve positive outcomes in assisting callers in their quitting attempts. ${ }^{4-6}$ Furthermore, these reactive telephone quitlines have also been demonstrated to represent quite an economical service. ${ }^{1}$ Studies have shown a clear relationship between mass media anti-smoking advertising and the number of calls to telephone quitlines, ${ }^{7}$ but the nature of relationships between the type of television advertisement and number of calls generated by particular placements of advertisements has received only limited attention. ${ }^{8}$ Yet this type of research has the potential to improve our understanding of how smokers respond to campaign advertising. ${ }^{1}$

Australia's national telephone Quitline has been promoted consistently throughout the National Tobacco Campaign (NTC) from May 1997 and has been supported by State and Territory government Quit campaign information providers and counsellors. One of the key objectives of this national service is to provide timely information and support to smokers to assist them in making a quit attempt. ${ }^{9}$ Incorporating the "call to action" of phoning the Quitline on all NTC television advertisements has provided smokers with the opportunity for a direct behavioural response to the evocative campaign advertising.

Miller et al $l^{9}$ reported that 144500 calls to the national Quitline were received within the first 12 months of its operation, including 123600 calls by smokers aged 18 years or older, representing approximately $4 \%$ of adult smokers. This proportion of smokers calling the Quitline is similar to the experience in the United Kingdom ${ }^{5}$ and in Scotland. ${ }^{10}$ In a separate study,
Miller et $a l^{9}$ examined the characteristics of callers to the national Quitline and reported that approximately 8 out of 10 callers were within the NTC target audience of 18-40 year olds, with similar proportions of males and females represented. Furthermore, callers expressed a positive response to their Quitline call and the 12 month point prevalence rate for successful quitting was $29 \%$. This suggests that although the majority of Australian smokers do not call the Quitline to assist them in their quitting attempt, for the substantial numbers of smokers who are sufficiently motivated to quit and do call the line, the experience seems to be associated with increased efficacy in quitting.

Some evidence is available about the process of generating calls to the Australian Quitline. For instance, a clear association between the media weight of campaign advertising and the generation of calls to the Quitline has been observed over the duration of the NTC to date. ${ }^{11-12}$ At the same time, differential sensitivities of call rates per advertising media weights have been noted between Australian States, ${ }^{11} 13$ thought to be caused by varying levels of Quitline promotion between States in previous years. In continuous information tracking studies over the campaign, ${ }^{13-14}$ the ability of NTC advertisements to generate calls to the Quitline was found to be influenced by an association between the executional characteristics of television advertisements and sensitivities to advertising media weight variations. Little is known, however, about what influence, if any, the type of programme a television advertisement is placed in has on its ability to generate Quitline calls.

Interestingly, although approximately one third of callers in Miller et al ${ }^{9}$ study reported that they had considered calling

Abbreviations: TARPs, Target Audience Rating Points; NTC, National Tobacco Campaign; WNTD, World No Tobacco Day 
Table 2 Comparison of performance of campaign advertisements in generating calls to the Quitline

\begin{tabular}{lrrrl}
\hline Commercial & $\begin{array}{l}\text { Advert } \\
\text { placements }\end{array}$ & $\begin{array}{l}\text { Total } \\
\text { TARPs }\end{array}$ & $\begin{array}{l}\text { Total } \\
\text { calls }\end{array}$ & $\begin{array}{l}\text { Ratio calls: } \\
\text { TARP }\end{array}$ \\
\hline Sydney & & & & \\
$\quad$ Tar & 43 & 353 & 276 & 0.78 \\
Eye & 56 & 301 & 284 & 0.94 \\
Call for help & 1 & 1 & 2 & 2.86 \\
Tar/call for help & 14 & 197 & 247 & 1.25 \\
$\quad$ Eye/call for help & 19 & 169 & 358 & 2.12 \\
Total & 133 & 1020 & 1168 & 1.14 \\
& & & & \\
Melbourne & & & & \\
Tar & 30 & 331 & 121 & 0.37 \\
$\quad$ Eye & 51 & 290 & 202 & 0.70 \\
Call for help & 2 & 1 & 1 & 0.91 \\
$\quad$ Tar/call for help & 9 & 164 & 100 & 0.61 \\
Eye/call for help & 13 & 162 & 178 & 1.09 \\
Total & 105 & 948 & 601 & 0.63 \\
\end{tabular}

Columns may not sum to the total figure due to rounding error.

one advertisement was broadcast within the hour prior to the call, the credit for that call was allocated proportionately to each advertisement placement within that hour. That is, if two campaign advertisements were broadcast within an hour prior to the call being made, each advertisement was credited with generating 0.5 calls. The total number of calls attributed to advertisements placed in each programme type was then divided by the number of TARPs achieved by those advertisements in order to calculate a calls per TARP rate.

Multiple regression analysis with binary variables was used to test the significance of differences between the efficiency of individual advertisements and combinations of advertisements and efficiency of placement in different programme types.

\section{RESULTS}

\section{Calls per TARP}

The relative performance of NTC advertisements in generating calls to the Quitline is shown in table 2. Calls per TARP ratios were consistently higher in Sydney than in Melbourne. Initially, comparing Sydney calls per TARP ratios for tar and eye as individual placements, eye generated a higher ratio of calls per TARP $(0.94)$ than did tar $(0.78)(p<0.01)$. The strategy of commencing ("topping") an advertisement break with tar and ending ("tailing") the break with call for help increased the rate of calls for this combination over the rate achieved by the placement of tar individually, from 0.78 to $1.25(p=0.02)$. Similarly, the combination of eye and call for help increased the ratio of calls per TARP compared with eye placed individually, from 0.94 to $2.12(\mathrm{p}=0.01)$.

The smaller number of advertisement placements in Melbourne yielded less conclusive results. As in Sydney, the calls per TARP ratio in Melbourne was higher for eye $(0.70)$ than for tar (0.37) when advertisements were placed individually $(\mathrm{p}<0.01)$. Although the combination of tar and call for help appears to have increased the calls per TARP ratio from 0.41 to 0.62 compared with the placement of tar individually, this difference was not significant $(p=0.19)$. Similarly, the difference in calls per TARP ratios between the combination of eye and call for help (1.09) and the individual placements of eye $(0.70)$ was not significant $(p=0.71)$.

\section{Programme type}

Table 3 illustrates the ratio of calls generated per TARP for each programme type in each city. Light entertainment, cultural/ informative, and reality TV programmes generated high calls per TARP ratios in both Sydney and Melbourne. Light entertainment programmes in particular yielded very high
Table 3 Programme type for Sydney and Melbourne in rank order

\begin{tabular}{lrrrl}
\hline Programme type & $\begin{array}{l}\text { Advert } \\
\text { placements }\end{array}$ & $\begin{array}{l}\text { Total } \\
\text { TARPS }\end{array}$ & $\begin{array}{l}\text { Total } \\
\text { calls }\end{array}$ & $\begin{array}{l}\text { Ratio calls: } \\
\text { TARP }\end{array}$ \\
\hline Sydney & & & & \\
Cultural/informative & 24 & 111 & 228 & 2.06 \\
Light entertainment & 9 & 20 & 41 & 2.05 \\
Reality TV & 7 & 93 & 136 & 1.47 \\
Drama & 22 & 195 & 278 & 1.42 \\
Inter programme & 1 & 17 & 22 & 1.25 \\
Sports & 9 & 38 & 38 & 1.02 \\
Comedy & 25 & 282 & 254 & 0.90 \\
Special & 1 & 13 & 9 & 0.70 \\
Movie & 18 & 181 & 118 & 0.65 \\
News/current affairs & 13 & 47 & 30 & 0.65 \\
Documentary & 4 & 24 & 15 & 0.61 \\
Total & 133 & 1020 & 1168 & 1.14 \\
& & & & \\
Melbourne & & & & \\
Light entertainment & 4 & 12 & 29 & 2.38 \\
Reality TV & 4 & 28 & 51 & 1.80 \\
Cultural/informative & 17 & 74 & 106 & 1.44 \\
Inter programme & 1 & 25 & 20 & 0.77 \\
News/current affairs & 7 & 20 & 14 & 0.70 \\
Sports & 10 & 47 & 28 & 0.58 \\
Drama & 19 & 192 & 107 & 0.55 \\
Comedy & 27 & 354 & 172 & 0.49 \\
Documentary & 2 & 12 & 5 & 0.42 \\
Movie & 14 & 183 & 71 & 0.39 \\
Total & 105 & 948 & 601 & 0.63 \\
\hline
\end{tabular}

Columns may not sum to the total figure due to rounding error.

ratios of calls per TARP for advertisements placed in these programmes in both cities (Sydney 2.05, Melbourne 2.38). In contrast, drama programmes rated quite efficiently in Sydney (1.42), but not in Melbourne (0.55). Documentaries and movies rated consistently among the least efficient programme types in both cities. With the low number of placements across the programme types, these differences were not statistically significant, and results should be viewed as suggestive only.

\section{DISCUSSION}

The observed higher rate of calls per TARP in Sydney compared with Melbourne is consistent with findings from earlier phases of NTC activity. ${ }^{13}$ This difference is not explained by the 9\% greater population of 16-39 year olds in Sydney ( 1.53 million) than in Melbourne ( 1.41 million), but probably reflects the lower level of previous campaign promotion of Quitline services in Sydney than in Melbourne, and hence greater sensitivity to offers for these services in Sydney.

Eye was found to have been more efficient in generating calls to the Quitline in both Sydney and Melbourne over this campaign period than was tar. Interestingly this finding contrasts with results from a continuous tracking study over the same period by Donovan and Jalleh, ${ }^{18}$ who found higher levels of advertising recall or "cut through" for tar than for eye. This raises some important questions around the particular characteristics of advertisements that generate action such as calling the Quitline. Although tar may be more easily recalled, possibly because it was building on residual awareness of another NTC advertisement ("lung"), or an even earlier antismoking advertisement ("sponge"), this recall may not necessarily reflect relative potential to generate direct action.

For instance, in tracking phase two of the NTC, Donovan ${ }^{13}$ noted that although calls to the Quitline increased when call for help was added to the campaign, levels of cut-through for the advertisement remained low. For the majority of smokers, the images of smoking, tar, and lungs may have been more easily recalled than the simulated damage to eye blood vessels. However, the dramatic image of the smoker's eye and the new information about the threat of impending blindness may 
have been more effective in driving calls to the Quitline among those at the stage where they were ready to take action to quit.

In essence, two separate advertising effects were being examined in the two studies, an initial effect to create awareness and influence attitudes toward smoking, and a second prompting the audience to take action. This distinction is often used in commercial advertising, where an advertiser may launch with a "brand building" campaign, and then later follow with a retail oriented "call to action" campaign. Within the overall NTC strategy, campaign communication activity is required to both encourage movement through stages of change toward quitting and to specifically prompt quitting action, such as calling the Quitline.

Combining a health effects execution with call for help increased the call rate for both tar and eye in Sydney. It is important to note that the calls per TARP calculations for the paired placements of health effect executions and call for help incorporate the combined TARPs achieved by both advertisements. Hence, the added efficiency achieved by the paired placements represents a synergy of advertising effect between the health effects advertisement and the modelling call for help, driving people to call the Quitline. In terms of seeking to achieve an objective of driving smokers to call the Quitline, the health effect advertisement could be seen as generating the motivation to take action now, while call for help facilitates the action of calling the Quitline by modelling the desired behavioural response. This rationale would be reasonable to apply to smokers who are at the stage of change toward quitting where they are ready to take action.

The calls per TARP efficiency rank order for programme types is very consistent for the top ranking types in the two cities. It should be noted that national commercial networking in Australia results in predominantly consistent programmes across the two cities. From the data examined in this study, it appears that light entertainment (games shows, variety shows), cultural/informative (information and infotainment shows), and reality TV programme types tended to be the most efficient for generating calls to the Quitline. These findings need to be viewed as preliminary, noting that the study period included only 13 placements in light entertainment programmes and 11 placements in reality TV. The findings for placements in cultural/informative programme types may be more robust, with 41 placements in these programmes being included in the analysis.

A key characteristic of the efficient cultural/informative and light entertainment programme types would be a relatively lower level of continuous involvement or engagement in content or storyline than in other types, such as movies, that rated as less efficient in generating Quitline calls. In this respect, however, it seems somewhat surprising that drama programmes rated relatively highly on the index in Sydney, although not in Melbourne. Because of the variety of programmes that comprise the reality TV programme type and the relatively small number of placements in these programmes, it is less clear how this programme environment may be achieving a reasonably high level of efficiency. The comedy programme type, where a reasonably large number of advertisements were placed, appears to be relatively inefficient in generating Quitline calls.

A number of limitations need to be considered when drawing conclusions from these results. The relatively short (seven week) period of campaign activity included in the analysis means that conclusions should be seen as preliminary, and analyses of further campaign advertising and Quitline call volume should be undertaken to confirm these findings.

The method of only attributing calls to advertisements that ran within an hour of the call being received ignores the cumulative impact of multiple exposures to the advertising. The measure of a person making a call within the hour reflects the final stage of taking action, but ignores the influence of earlier exposures to this and to other NTC advertisements.
Consistent with the notion of a cumulative effect of campaign exposure, Donovan and Jalleh ${ }^{18}$ found that measures of smokers thinking about quitting several times a day increased steadily over the course of this campaign phase. This bias would serve to increase the calls per TARP ratio in favour of individual placements of eye, which ran in weeks four and five, compared with individual placements of tar, which ran in weeks one, two and three, and would further advantage the combination placements of health effect advertisements and call for help, which occurred almost exclusively in week seven. Research should be undertaken over campaign periods with different rotation of advertisements in order to clarify these effects.

Another potential confounder in the study is the length of the programmes in each programme type. Movies, for instance, tend to be longer programmes than the light entertainment type or the infotainment shows of the cultural/ informative type, and the method of only including in the analysis calls within one hour of an advertisement being broadcast could possibly reflect a bias toward shorter programmes. This would only be the case if smokers waited until after the end of a programme to make their call to the Quitline. The experience from direct marketing campaigns, however, suggests that if someone is going to make a call in response to a television advertisement, he or she will act relatively soon after seeing the advertisement rather than waiting until the end of a programme. ${ }^{16}$ Further research with callers to the Quitline could help clarify this issue.

In addition, it is possible that the time of day that particular programme types are on television may act as a confounder in assessing the efficiency of programmes to generate calls. This also warrants investigation in further studies of this type.

\section{CONCLUSION}

The limitations noted above mean that caution should be exercised in drawing definitive conclusions until further studies are undertaken with larger numbers of advertisements. Nevertheless, on the basis of data examined in this study, it appears that the eye advertisement with its new information and high level threat of the risk of blindness has been more efficient in generating calls to the Quitline than the tar advertisement with its already more accepted message about tar in a smoker's lungs. This provides evidence about the ability of advertisements portraying new health information to smokers in a visually graphic way to generate Quitline calls. The study suggests that the creative strategy of modelling calls to the Quitline in call for help appears to add synergistically to the impact of the health effects advertisements when paired with them in the same advertising break. In order to achieve the objective of generating Quitline calls, this study suggests media planners should place campaign advertisements in the lower involvement programme types of light entertainment, informational/infotainment programmes, and reality TV. Further research over subsequent campaign phases should be undertaken to assess how confidently these findings can be generalised. Planning for campaign media buys needs to consider both the strategic requirement of driving smokers through stages of change toward quitting as well as generating specific quitting action among those ready to quit.

\section{ACKNOWLEDGEMENTS}

Funding for this study was provided by the Australian Department of Health and Ageing. The authors would like to acknowledge the assistance of Mr Charlie Nelson from Foreseechange in providing statistical support for the project.

\section{Authors' affiliations}

T Carroll, The Australian Department of Health and Ageing, Sydney B Rock Mitchell Media, Sydney 


\section{REFERENCES}

1 Wakefield $M$, Borland R. Saved by the bell: the role of telephone helpline services in the context of mass-media anti-smoking campaigns. Tob Control 2000;9:1 17-9.

2 Owen N, Roberts L, Wakefield M. The South Australian Quit Advisory Service: characteristics of callers and cessation outcomes. Health Promotion Journal of Australia 1995:5:49-50.

3 Balanda KP, Lowe JB, O'Connor-Fleming M-L. Comparison of two self-help smoking cessation booklets. Tob Control 1999;8:57-61.

4 Zhu S-H, Anderson CM, Johnson CE, et al. A centralised telephone service for tobacco cessation: the California experience. Tob Control 2000:9(Suppl II):ii 48-55.

5 Owen L. Impact of a telephone helpline for smokers who called during a mass media campaign. Tob Control 2000;9:148-54.

6 Zhu SH, Anderson CM, Tedeschi GJ, et al. Evidence of real-world effectiveness of a telephone quitline for smokers. New Engl J Med 2002; 347: 1087-93.

7 Pierce JP, Anderson DM, Romano RM, et al. Promoting smoking cessation in the United States: effect of public service announcements on the Cancer Information Service telephone line. J Natl Cancer Inst 1992;84:677-83.

8 Powers $\mathbf{P}$, Ranger-Moore J, Wentzel TM, et al. Impact of television advertising on helpline client's stages of change. Sixth Annual Scientific sessions of the Society for Nicotine and Tobacco Research, Arlington Virginia, USA, February 18-20, 2000. www.srnt.org/events/ abstracts $00 /$ index.htm

9 Miller CL, Wakefield M, Roberts L. Uptake and effectiveness of the Australian telephone Quitline service in the context of a massmedia campaign. Tob Control 12(Suppl II);ii53-8.
10 Platt S, Tannahill A Watson J, et al. Effectiveness of antismoking telephone helpline: follow up survey. BN 1997;314:137.

11 Williams $\mathbf{P}$, Bleasdale T. The relationship between campaign television activity and quitline call data. In: Hassard K, ed. Australia's National Tobacco Campaign: evaluation report volume one. Canberra:

Commonwealth Department of Health and Aged Care, 1999;107-26.

12 Tan N, Hassard K. Quitline calls and campaign television investment. In: Hassard K, ed. Australia's National Tobacco Campaign evaluation: report volume two. Canberra: Commonwealth Department of Health and Aged Care, 2000;133-8.

13 Donovan, R. Tracking the National Tobacco Campaign phase two. In Hassard K, ed. Australia's National Tobacco Campaign evaluation: report volume two. Canberra: Commonwealth Department of Health and Aged Care, 2000; 1 15-32.

14 Donovan R, Freeman J, Borland R, et al. Tracking the National Tobacco Campaign. In: Hassard K, ed. Australia's National Tobacco Campaign evaluation: report volume one. Canberra: Commonwealth Department of Health and Aged Care, 1999; 127-65.

15 www.oztam.com.au/discoveryguide/images/termsanddefinitions.pdf

16 Pragerl. The media: new opportunities, different criteria. Why DM planning has its own rules, and what these demand. AdMap July, 1993:37-9

17 Nielsen AC. Metropolitan Television Media Adviser. Training Manual Version 2.3. Sydney: AC Nielsen, 2000.

18 Donovan RJ, Jalleh G. Tracking the 2000 National Tobacco Campaign In: Australia's National Tobacco Campaign: evaluation report volume three. Canberra: Commonwealth Department of Health and Ageing. In press. 